\title{
Detection of Gad-Enhancing Lesions in Multiple Sclerosis Using Conditional Random Fields
}

\author{
Zahra Karimaghaloo ${ }^{1}$, Mohak Shah ${ }^{1,3}$, Simon J. Francis ${ }^{2}$, Douglas L. Arnold ${ }^{3}$, \\ D. Louis Collins ${ }^{2}$, and Tal Arbel ${ }^{1}$ \\ ${ }^{1}$ Centre for Intelligent Machines, McGill University, Canada \\ ${ }^{2}$ Montreal Neurological Institute, McGill University, Canada \\ ${ }^{3}$ NeuroRx Research, Montreal, Canada *
}

\begin{abstract}
Identification of Gad-enhancing lesions is of great interest in Multiple Sclerosis (MS) disease since they are associated with disease activity. Current techniques for detecting Gad-enhancing lesions use a contrast agent (Gadolinium) which is administered intravenously to highlight Gad-enhancing lesions. However, the contrast agent not only highlights these lesions, but also causes other tissues (e.g. blood vessels) or noise in the Magnetic Resonance Image (MRI) to appear hyperintense. Discrimination of enhanced lesions from other enhanced structures is particularly challenging as these lesions are typically small and can be found in close proximity to vessels. We present a new approach to address the segmentation of Gad-enhancing MS lesions using Conditional Random Fields (CRF). CRF performs the classification by simultaneously incorporating the spatial dependencies of data and labels. The performance of the CRF classifier on 20 clinical data sets shows promising results in successfully capturing all Gad-enhancing lesions. Furthermore, the quantitative results of the CRF classifier indicate a reduction in the False Positive (FP) rate by an average factor of 5.8 when comparing to Linear Discriminant Analysis (LDA) and 1.6 comparing to a Markov Random Field (MRF) classifier.
\end{abstract}

\section{Introduction}

Multiple Sclerosis (MS) is a disorder of the central nervous system and is a common disease among young adults. Magnetic Resonance Imaging (MRI) is widely used to study this disease and assess its temporal progress. In particular, MRI is well established as the optimal imaging technique for detecting MS lesions which are believed to be highly correlated with the presence of disease activity in the absence of clinical changes. Moreover, using a contrast agent (e.g. Gadolinium) with T1-weighted (T1w) imaging can help to identify areas which represent the initial stage of lesion development. It is generally believed that the number and volume of enhancing lesions are important indicators of disease activity in MS. These indicators have generally been determined by using operator guided segmentation methods. However, in addition to being laborious and time

\footnotetext{
^ This work was supported by NSERC Strategic Grant (350547-07).
} 
consuming, manual detection is prone to intra - and - inter expert variability making the analysis of the results very complicated. Therefore, it is desirable to have a fully automatic segmentation scheme for detection of enhanced lesions in studies with large ensemble of patient data. Unfortunately, automatic identification of enhanced lesions is a challenging task due to the presence of many non-lesion enhancements, which appear as small regions and are very similar to Gad-enhancing lesions (unlike T2-weighted lesions, Gad-enhancing lesions are usually very small).

Even though there have been many studies for MS lesion detection on T2weighted (T2w) MR images, only a few have investigated assessment of enhanced lesion detection. Bedell et al. [1] suggested using a special pulse sequence for T1w with contrast (T1c) imaging which increases the contrast between blood signal enhancement and lesion enhancement. In addition to the need for a special pulse sequence, this algorithm also requires user inputs for initial seed placement for detection of cerebro-spinal fluid to eliminate enhanced areas caused by circumventricular organs particularly in the region of choroid plexus. Miki et al. 2] proposed using a fuzzy connectivity to delineate enhanced lesions. Their approach is not fully automatic, as it requires human confirmation after each enhanced area is found by the algorithm. The study by Narayana et al. 3 also uses special pulse sequence as in 11. Although it is a fully automatic approach, it requires prior segmentation of $\mathrm{T} 2 \mathrm{w}$ lesions. In this paper, we propose a novel fully automatic probabilistic technique which is one step towards a clinical tool for detection of enhanced lesions. Our framework does not need human intervention and also uses only the commonly acquired MRI sequences (i.e T1w before and after contrast, T2w and FLAIR). Furthermore, our approach does not rely on pre-segmentation of $\mathrm{T} 2 \mathrm{w}$ lesions in other sequences. We model the enhanced lesion identification problem as a probabilistic classification task in which for each instance (i.e. pixel in the MRI) we seek to find the best labeling. This is achieved by computing the posterior distribution over the joint distribution of labels given the observations. A deterministic decision can be sought afterwards in order to have a binary classification result.

Our framework uses a discriminative model based on Conditional Random Fields (CRFs) 415] to model the posterior distribution of labels. There are two advantages to CRF-based models. First, they directly model the posterior distribution, and hence avoid modeling the joint distribution of observations and labels. Modeling the joint distribution is a complicated and expensive task and often leads to simplifying assumptions that may not generally be valid (especially in the field of medical imaging). The second benefit of a CRF-based model is that the the spatial dependencies among instances can be modeled in a principled manner [5]. In order to model these dependencies, both labels of neighbouring instances, as well as their observations are taken in to account. This is in contrast to traditional approaches for modelling data dependencies (e.g. MRF), where typically only the neighbouring labels are considered. Although consideration of label dependencies may be sufficient in some applications, incorporating observation dependencies provides a more powerful source of information that can be 
exploited to achieve better classification results. Particularly, in the case of enhanced lesion identification, many False Positives (FPs) which occur due to the enhancement of vascular structures can be avoided by incorporating the neighbouring observations. The experimental results of applying the CRF classifier on a multi-centre clinical data sets from patients with various stages of relapsing remitting MS show significant improvements over traditional classification approaches.

The remains of this paper is organized as follows: Section 2 reviews the background of the CRF and elaborates on our CRF-based classifier. We then present the classification results of Gad-enhanced lesions in Section 3. Section 4 summarizes the paper and discusses the possibilities for further developments.

\section{Method}

Traditional classification techniques such as Decision Trees, Linear Discriminant Analysis (LDA) and Support Vector Machines (SVM) assume data instances are independent and identically distributed. While this assumption is valid for many data mining problems, in the context of image region labeling, it is important to incorporate contextual information in the form of spatial dependencies between pixels in the image. Since labels of neighbouring pixels are typically correlated, integration of spatial information generally yields smoother and more reliable results.

Markov Random Fields (MRF) have been extensively used to capture contextual constraints by allowing the classification of one instance to depend on the labels of neighbouring instances. In fact, MRF is a generative approach which models the joint probability distribution of the image observations and their corresponding labels. Letting $\mathbf{x}_{i} \in \Re^{d}$ and $y_{i} \in\{1,0\}$ show the observation vector and the label of the $i^{\text {th }}$ instance respectively, MRF models the posterior distribution over all labels $\mathbf{Y}=\left\{y_{1}, \ldots, y_{n}\right\}$ given all observations $\mathbf{X}=\left\{\mathbf{x}_{1}, \ldots, \mathbf{x}_{n}\right\}$ as:

$$
p(\mathbf{Y} \mid \mathbf{X}) \propto p(\mathbf{X}, \mathbf{Y})=p(\mathbf{X} \mid \mathbf{Y}) p(\mathbf{Y})
$$

The priori distribution, $p(\mathbf{Y})$, is commonly modeled as a Markov field based on the neighbouring labels. Since this prior does not depend on the observations, the label interactions are modeled neglecting the observed data. This may lead to over smoothing of regions. Furthermore, for tractability purposes, the observations are assumed to be independent given the labels. Thus the likelihood can be expressed with a simple factorized form of $p(\mathbf{X} \mid \mathbf{Y})=\Pi_{i} p\left(\mathbf{x}_{i} \mid y_{i}\right)$. As a result of this restrictive independence assumption, the interactions among observations are not generally modeled in MRF.

Many discriminative approaches such as CRF [4, and its derivatives (Discriminant Random Field (DRF) [5] and Support Vector Random Field (SVRF) [6]) have been proposed to overcome shortcomings of MRF by integrating dependencies among observations into the model. In a discriminative framework, we directly model the posterior distribution $p(\mathbf{Y} \mid \mathbf{X})$ without building the joint distribution. Particularly, discriminative approaches relax the conditional independence 
assumption of the observations and allow modeling of observation dependent interactions. Incorporating the neighbouring observations should help achieve better classification results. Specifically, in the context of enhanced lesion identification, many FPs can be excluded, due to the fact that many vascular structures (especially those within CSF) have different neighbourhood observations than enhanced-lesions.

As graphical models are widely used to represent probabilistic relationships between instances, we continue using graphical notations to explain the formulation of discriminative methods. In a graphical model $G(V ; E)$, instances are shown with a set of nodes (V) and their conditional (in)dependencies are represented by (missing)edges (E). Inspired by the model suggested by Lafferty et al. 4, CRF-based models formulate the posterior probability of labels given the observations as:

$$
p(\mathbf{Y} \mid \mathbf{X})=\frac{1}{Z} \Pi_{i \in V} \phi_{i}\left(y_{i} \mid \mathbf{X}\right) \Pi_{i, j \in E} \psi_{i, j}\left(y_{i}, y_{j} \mid \mathbf{X}\right)
$$

where $Z$ is the normalization term and $\phi_{i}$ is the "association" potential which models dependencies between the label of the $i^{\text {th }}$ node, $y_{i}$, and the set of all (or a part of) observations $\mathbf{X} . \psi_{i, j}$, is the "interaction" potential which represents dependencies between the labels of neighbouring nodes given the observations $\mathbf{X}$. As it can be observed from the above expression, an important difference between the prior distribution in MRF and the "interaction" potential in CRF is the incorporation of observations for modeling the neighbourhood interactions of labels. This implies that CRF can optimally learn the interaction of adjacent labels based on their observations. One immediate advantage of this is to modify the smoothness among labels by taking into account the observed data and therefore prevent over smoothing [5]. Moreover, we notice that observations are not considered independent in the "association" term, in contrast to the conditional independent assumption for observations that is usually made in MRF models. In the following section we elaborate on how we model $\phi_{i}$ and $\psi_{i, j}$ for the problem of enhanced lesion detection.

\subsection{Association Potential}

Similar to previous work [5]7 we use LDA to capture the interactions of the label of each instance and the observations. Even though $\phi_{i}$ can be a function of all the observations in principle, to keep the model simple, we assume that only the observations at each instance are included in the association potential [78. Therefore, the association potential can be written as:

$$
\phi_{i}\left(y_{i}=1 \mid \mathbf{x}_{i}\right)=\sigma\left(\mathbf{w}^{T} \mathbf{x}_{i}\right)=\frac{1}{1+\exp \left(-\mathbf{w}^{T} \mathbf{x}_{i}\right)}
$$

where $\mathbf{w}$ is the "association" parameter vector which is obtained in the training phase (Section 2.3). 


\subsection{Interaction Potential}

The "interaction" potential in CRF allows for learning the relationships between the neighbouring labels given the observed data. Accordingly, we define observation dependent terms for adjacent instances and use a set of parameters to modulate the coupling between the labels of adjacent instances as well as their observed data. In particular, we use the following expression for the interaction potential:

$$
\psi_{i, j}\left(y_{i}, y_{j} \mid \mathbf{x}\right)=\exp \left(y_{i}, y_{j} \theta^{T} \mathbf{F}_{i, j}\right)
$$

where $\theta$ is the "interaction" parameter vector obtained in the training phase (Section 2.3). $\mathbf{F}_{i, j}=\left[f\left(\mathbf{x}_{i}, \mathbf{x}_{j}\right) 1\right]$ consists of a data dependent term, $f\left(\mathbf{x}_{i}, \mathbf{x}_{j}\right)$, as well as a constant. The data-dependent term computes the similarity of adjacent observations to modulate the smoothness over neighbouring instances accordingly. Moreover, the constant term takes into account the interaction of adjacent labels.

\subsection{Parameter Learning and Inference}

Simultaneously determining the optimal parameters of the "association" potential and the "interaction" potential can be performed numerically as a convex optimization problem. The parameters of the model $\boldsymbol{\Theta}=\{\mathbf{w}, \theta\}$ are learned in the training phase within a maximum likelihood framework as follows:

$$
\begin{aligned}
L(\boldsymbol{\Theta}) & =\log p(\mathbf{Y} \mid \mathbf{X}, \boldsymbol{\Theta}) \\
& =\Sigma_{m=1}^{M}\left[\left(\Sigma_{i \epsilon V} \phi_{i}\left(y_{i} \mid \mathbf{X}^{m}\right)+\Sigma_{i, j \epsilon E} \psi_{i, j}\left(y_{i}, y_{j} \mid \mathbf{X}^{m}\right)\right)-\log Z_{\mathbf{x}^{m}}\right] \\
\boldsymbol{\Theta}^{*} & =\arg \max _{\boldsymbol{\Theta}} L(\boldsymbol{\Theta})
\end{aligned}
$$

with $M$ being the total number of training sets. Note that since the observation is not modeled, the normalization term is a function of observations $X^{m}$ for each training set $m$. Once the parameters are learned during training, the problem of classification is to find the most probable set of labels $\mathbf{Y}^{*}$.

$$
\mathbf{Y}^{*}=\arg \max _{\mathbf{Y}} p(\mathbf{Y} \mid \mathbf{X}, \boldsymbol{\Theta})
$$

The inference problem can be solved with different approaches (e.g. iterated conditional modes, graph cuts, belief propagation technique). We adapted a loopy belief propagation algorithm [9] to solve this problem.

\section{$3 \quad$ Experiments and Results}

\subsection{Data Pre-processing}

We evaluate the performance of the CRF classifier on a multi-centre clinical data set consisting of 25 multidimensional MRI volumes. This data set is acquired from patients with relapsing remitting (RR) MS, all having a heavy load of Gad-enhancing lesions (9 on average). Each acquisition is composed of three 
sequences: T1w (before and after contrast) T2w and FLAIR. All volumes are $3 \mathrm{~mm}$ thick axial slices with $1 \mathrm{~mm} \times 1 \mathrm{~mm}$ intra-plane resolution. For each patient, the Gad-enhancing lesions were manually labeled by 3 experts resulting in a silver standard where the "ground truth" lesion pixels result from a consensus among the experts. Prior to classification, MRI data needs to be preprocessed in order to correct for imaging artifacts. These pre-processing steps include bias-field inhomogeneity correction using N3 [10] as well as removal of non-brain regions from the MRI [1]. Furthermore, the intra-subject sequences are registered to their T1-w space [12] and the intensity range of all sequences is normalized [13].

The CRF classifier is trained on $5 \mathrm{MRI}$ volumes and tested on the remaining 20 and its performance is compared against LDA and MRF classifiers. The LDA classifier is defined as in Eq[3] which only includes the "association" potential (i.e. no spatial dependency is captured). Our MRF classifier is formulated similar to $\mathrm{CRF}(\mathrm{Eq} 2)$ where the only difference is that the "interaction" potential (Eq 4) is modified to be only dependent on the labels (i.e., $f\left(\mathbf{x}_{i}, \mathbf{x}_{j}\right)$ is removed). With this definition, we highlight the improvements of CRF which are gained due to incorporation of the data dependent term.

\subsection{Qualitative Results}

Figure 1 illustrates two qualitative examples of the classifier results. In each row we show: (a) a T1c slice along with the classification results of the three classifiers: (b) LDA, (c) MRF and (d) CRF. In each case, green and red colors represent True Positives (TP) and FPs, respectively. The selected slice in the first row has enhanced structures that are both lesions and non-lesions while the example in the second row only has a heavy load of non-lesion enhanced structures. As it can be seen, all three classifiers were able to capture enhanced lesions (TP). However, CRF outperforms LDA and MRF in successfully deleting all the FPs by using the neighbourhood intensity information (in addition to the label information considered by MRF).

\subsection{Quantitative Results}

The performance of CRF is quantitatively evaluated against LDA and MRF. To that end, the TPs and FPs are defined as follows: if more than $50 \%$ of a lesion is captured, the region is counted as a TP, and any falsely detected region with area greater than two pixels is considered a FP. The quantitative results showed that all 141 Gad-enhancing lesions in the cohort of 20 test volumes were detected by all three classification methods. The absolute number of FPs per each patient is depicted in Figure 2(a). Comparing the results, we note that CRF outputs significantly less FPs. In fact, the FP rate in the CRF results is reduced by an average factor of 5.8 and 1.6 upon comparing to LDA and MRF, respectively. It should be noted that many of the FPs, especially in the $\mathrm{CRF}$ and MRF results are regions with only two pixels. Due to the presence of Gad-enhancing lesions with an area of two pixels, no further post-processing was applied to the classification results to eliminate FPs with small areas. Moreover, comparison of the dice coefficient (Figure 2(b)) for the three classifiers suggests 

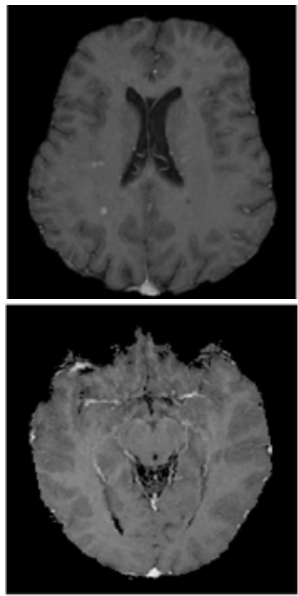

(a)
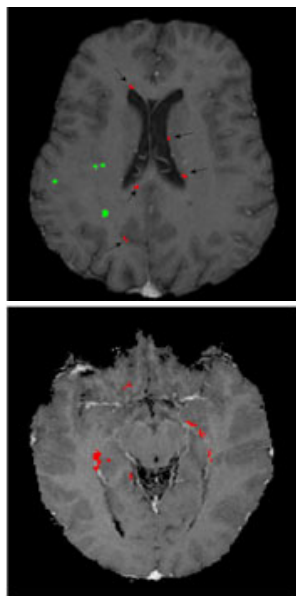

(b)
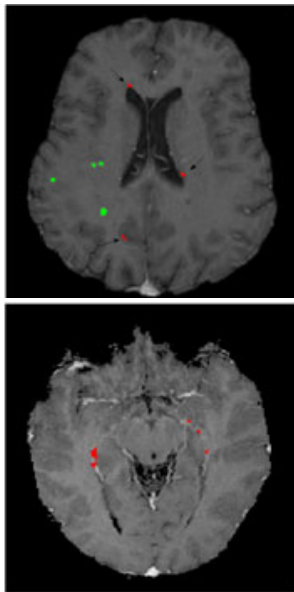

(c)
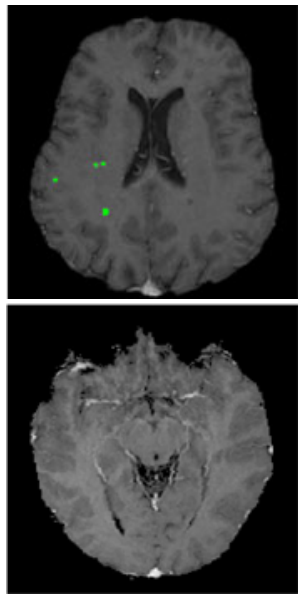

(d)

Fig. 1. Two examples of the classification results. (a) T1w image, (b), (c) and (d) classification results for LDA, MRF and CRF respectively. TPs and FPs are shown in green and red, respectively.

an improvement by a factor of 2.1 and 1.3 over LDA and MRF, respectively. However, it should be noted that since Gad-enhancing lesions are very sparse and small, dice coefficient is not suited as a measure of choice for evaluation. This is observed in cases 3, 4, 6, 9 and 17 where low values are observed even though the algorithm performs acceptably in terms of TP and FP. Therefore, TP and FP statistics present a more reasonable measure on classifier performance.

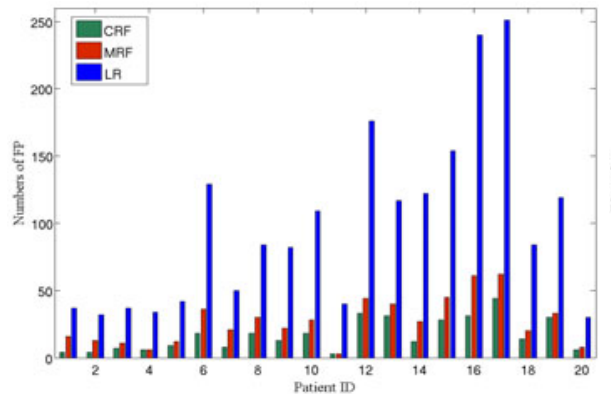

(a)

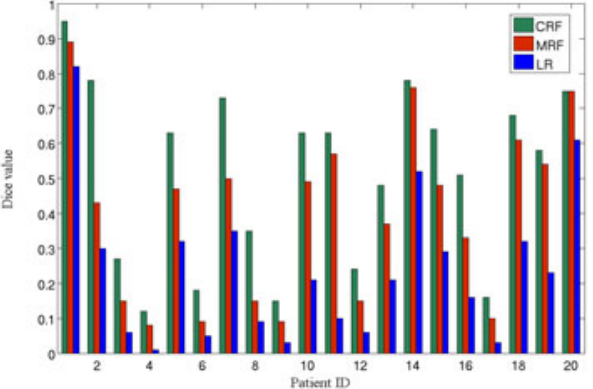

(b)

Fig. 2. (a) Numbers of FPs and (b) Dice value for the 20 patients in all three methods

\section{Discussion}

In this paper we propose a new approach to address the problem of enhanced lesion segmentation in MRI. Our framework uses a CRF-based classification technique to model the spatial relations of the data in a principled manner. Unlike commonly used MRF approaches, where only spatial dependencies of adjacent labels are modeled, the CRF classifier learns the interactions among neighbouring 
instances by simultaneously considering their labels and observations. Our classifier is validated on 20 multi-centre real clinical data set from RR MS patients with varying loads of Gad-enhancing lesions. Since there are many non-lesion enhanced structures in the T1c image, excluding FPs is of critical importance in this problem. The experimental results show the advantage of incorporating data spatial dependencies particularly to reduce the number of FPs by an average factor of 1.6 compared to MRF. However, in the current model, both "interaction" and "association" potentials are assumed to have simplified linear models. In the future, we will exploit more sophisticated models to increase the discrimination power of the classifier, by considering shape-based neighbourhood information (inspired by the fact that most vascular structures have line-like shapes). Furthermore, we would also like to investigate the performance of the CRF classifier for the more general problem of lesion segmentation in T2w MRI.

\section{References}

1. Bedell, B., Narayana, P.: Automatic segmentation of Gadolinium-enhanced multiple sclerosis lesions. Magn. Reson. Med 39, 935-940 (1998)

2. Miki, Y., Grossman, R., Udupa, J., Samarasekera, S., van Buchem, M., Cooney, B., Pollack, S., Kolson, D., Constantinescu, C., Polansky, M., Manon, L.J.: ComputerAssisted Quantitation of Enhancing Lesions in Multiple Sclerosis: Correlation with Clinical Classification. American Journal of Neuroradiology 18, 705-710 (1997)

3. He, R., Narayana, P.: Automatic delineation of Gd enhancements on magnetic resonance images in multiple sclerosis. Med. Phys. 29, 1536-1546 (2002)

4. Lafferty, J., McCallum, A., Pereira, F.: Conditional Random Fields: Probabilistic Models for Segmenting and Labeling Sequence Data. In: 18th Int. Conf. on Machine Learning, pp. 282-289 (2001)

5. Kumar, S., Hebert, M.: Discriminative Random Fields. International Journal of Computer Vision, 179-201 (2006)

6. Lee, C., Greiner, R., Schmidt, M.: Support vector random fields for spatial classification. In: Jorge, A.M., Torgo, L., Brazdil, P.B., Camacho, R., Gama, J. (eds.) PKDD 2005. LNCS (LNAI), vol. 3721, pp. 121-132. Springer, Heidelberg (2005)

7. Lee, C., Wang, S., Murtha, A., Brown, M., Greiner, R.: Segmenting Brain Tumors Using Pseudo-Conditional Random Fields. In: Metaxas, D., Axel, L., Fichtinger, G., Székely, G. (eds.) MICCAI 2008, Part I. LNCS, vol. 5241, pp. 359-366. Springer, Heidelberg (2008)

8. Plath, N., Toussaint, M., Nakajima, S.: Multi-class image segmentation using Conditional Random Fields and Global Classification. In: Int. Conf. on Machine Learning (2009)

9. Murphy, K., Weiss, Y., Jordan, M.: Loopy Belief Propagation for Approximate Inference: An Empirical Study. In: Foo, N.Y. (ed.) AI 1999. LNCS, vol. 1747, pp. 467-475. Springer, Heidelberg (1999)

10. Sled, J., Pike, B.: Correction for $\mathrm{b}(1)$ and $\mathrm{b}(0)$ variations in quantitative $\mathrm{t}(2)$ measurements using MRI. Mag. Reson. Med. 43, 589-593 (2000)

11. Smity, S.: Fast Robust automated brain extraction. Hum. Brain Mapp. 17, 143-155 (2002)

12. Collins, D., Neelin, P., Peters, T., Evans, A.: Automatic 3d intersubject registration of MR volumetric data in standardised Talairach space. Comp. Assisted Tomography 18, 192-205 (1994)

13. Nyul, L., Udupa, J.: On standardizing the MR Image Intensity Scale. Comp. Assisted Tomography 42, 1072-1081 (1999) 